УДК 87.35 .91

\title{
АНАЛИЗ ЭФФЕКТИВНОСТИ ПРИМЕНЕНИЯ САЙКЛИНГ-ТЕХНОЛОГИИ ПРИ РАЗРАБОТКЕ ЗАЛЕЖЕЙ ГАЗОВОГО КОНДЕНСАТА
}

\author{
Волженина Диана Алексеевна1, \\ Volzhenina_DA@irkutskoil.ru
}

\section{Шарф Ирина Валерьевна ${ }^{1}$,} irina_sharf@mail.ru

\author{
Сабанчин Игорь Валентинович2, \\ isabanchin@irkutskoil.ru \\ 1 Национальный исследовательский Томский политехнический университет, \\ Россия, 634050, г. Томск, пр. Ленина, 30. \\ 2 Иркутская Нефтянная Компания, \\ Россия, 664007, г. Иркутск, пр. Большой Линейный, 4.
}

\begin{abstract}
Актуальность исследования обусловлена тем, что при разработке газоконденсатных залежей на месторождениях нефрти и газа наблюдаются процессы ретроградной конденсации, вследствие которых в пласте безвозвратно теряются значительные объемы высококипящих компонентов уелеводородной группы С5+, добыча которых являлась бы дополнительным доходным источником для нефтегазовых компаний. Общие запасы газового конденсата в России составляют около 2 млрд m, поэтому для решения проблемы наиболее полного извлечения компонентов газоконденсатной пластовой смеси необходимо внедрение новых эфффективных методов добычи.

Целью исследования является обоснование эффрективности применения сайклинг-технологии для разработки газоконденсатной залежи.

Объект: месторождение N, расположенное на территории Восточной Сибири.

Методы: композиционное, геологическое и гидродинамическое моделирование газоконденсатной залежи

Результаты. Проведено композиционное моделирование газоконденсатной пластовой смеси в соответствии с компонентным составом проб газа и газового конденсата, а также результатами газоконденсатных исследований. Построена упрощенная геологическая модель исследуемой залежи, на основе которой проведено гидродинамическое моделирование ее состояния на различных режимах разработки. Сравнительный анализ показателей разработки газоконденсатной залежи, таких как коэффициент извлечения конденсата, коэфициент извлечения компонентов $\mathrm{C}_{2}-\mathrm{C}_{4}$ и конденсато-газовый фрактор, на различных режимах работы доказал, что применение сайклинг-технологии приводит к увеличению извлечения компонентов уәлеводородной группы $C_{5+}$ и позволяет решать ряд экономических и экологических задач. Возможна адаптация представленной модели под необходимые горно-геологические условия с целью применения данной технологии на территории ЗападноСибирской и других нефтегазоносных провинций.
\end{abstract}

\section{Ключевые слова:}

Месторождение, разработка, высококипящие группы углеводородов, сайклинг-технология, газоконденсатная залежь, композиционное, геологическое и гидродинамическое моделирование, коэфффициент извлечения конденсата.

\section{Введение}

Основной особенностью залежей газового конденсата является большое содержание в нем углеводородов группы $\mathrm{C}_{5+}$, которые при изотермическом снижении давления выпадают в жидкую фазу. Такой процесс называется ретроградной конденсацией и ведет не только к потерям высококипящих углеводородов, являющихся значимым источником доходов для добывающих компаний, но и к осложнениям в процессе разработки. В связи с этим газоконденсатные системы были признаны типом резервуара с наиболее сложными термодинамическими характеристиками и поведением газожидкостного потока.

Фазовые превращения газового конденсата с изменением температуры и давления происходят поэтапно. При начальном пластовом давлении $\left(P_{\text {пл}}\right)$ смесь находится в газообразном состоянии, однако с началом разработки давление в пласте начинает снижаться и при достижении давления, соответствующего уровню начала конденсации $\left(P_{\text {нк}}\right)$, смесь переходит в двухфазное парожидкостное состояние. Доля жидкой фазы растет вплоть до достижения давления максимальной конденсации, после чего при дальнейшем снижении давления жидкая фаза испаряется. Однако немаловажно то, что переход конденсата из жидкой фазы в газовую происходит при низких давлениях, которые не достигаются при разработке месторождений и на практике обратного испарения газового конденсата в полной мере не наблюдается. Кроме того, в пористой среде испарению выпавшей жидкой фазы могут препятствовать силы взаимодействия с поверхностью породы [1].

Газожидкостные потоки в ретроградных газоконденсатных залежах можно разделить на три области (рис. 1).

1. Первая область находится на удалении от призабойной зоны, и Рпл в ней выше Рнк, в этой области присутствует только однофазный углеводородный газ.

2. Вторая область - область накопления конденсата, где жидкость выпадает из газовой фазы, но еще не 
является подвижной, то есть поток газа все еще является однофазным.

3. Третья область, или призабойная зона пласта, отличается более низким давлением, чем в предыдущих зонах. Таким образом, насыщение жидкостью увеличивается, что обуславливает течение к стволу скважины конденсата не только в газовой, но и в жидкой фазе, что приводит к значительному снижению относительной проницаемости по газу и, тем самым, уменьшается продуктивность добывающей скважины [2, 3].

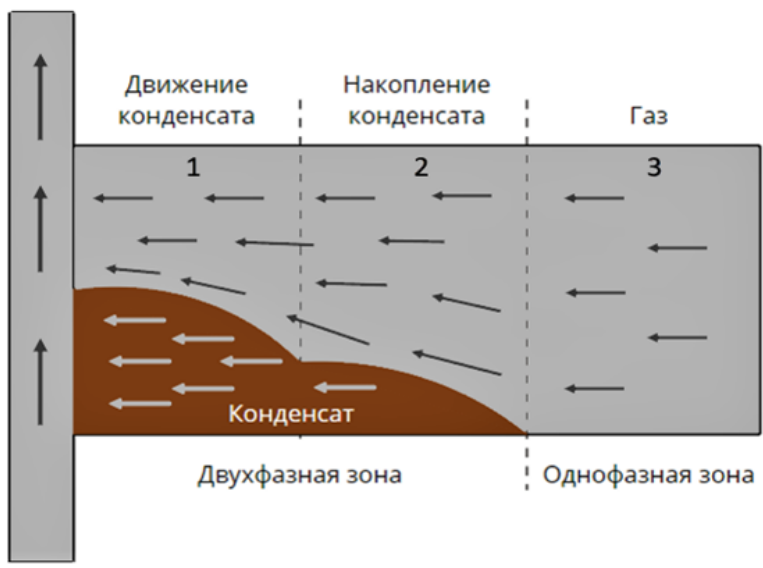

Pис. 1. Газожидкостные потоки в газоконденсатных залежах

Fig. 1. Gas-liquid flows in gas-condensate reservoir

По оценкам ИНГГ СО РАН общие запасы газового конденсата в России составляют около 2 млрд т, поэтому проблема наиболее полного извлечения компонентов газоконденсатной пластовой смеси, содержащейся в залежах газового конденсата, является актуальной для нефтегазодобывающих компаний и требует внедрения новых эффективных методов добычи [4-6]. Одним из подходов к разработке таких залежей является применение сайклинг-технологии, которая заключается в поддержании пластового давления путем нагнетания в продуктивный горизонт сухого газа. Целью применения данной технологии является предотвращение ретроградной конденсации жирного углеводородного газа в призабойной зоне пласта, увеличение коэффициента извлечения конденсата и получение газового конденсата в качестве товарного продукта. Помимо этого, становится возможным решить экологическую проблему, связанную с утилизацией попутного газа в случае отсутствия инфраструктуры для его подготовки и сбыта, путем консервации запасов газа до возможности их реализации $[7,8]$. Таким образом, целью данного исследования является обоснование эффективности применения сайклинг-технологии при разработке газоконденсатной залежи на примере месторождения $\mathrm{N}$ Восточной Сибири и возможного тиражирования данной технологии на месторождениях Западной Сибири.

\section{Материалы и методы}

Обоснование эффективности применения сайклинг-технологии (далее сайклинг) на газоконден- сатных месторождениях проводилось путем сравнительного анализа технологических показателей, полученных для различных режимов разработки. Основным инструментом проведения сравнительного анализа являются следующие виды моделирования:

1) композиционное;

2) геологическое;

3) гидродинамическое.

\section{Результать}

Композиционное моделирование.

Создание модели флюида

Наиболее доступным и эффективным средством контроля за пластовой системой, а также инструментом визуализации процессов, происходящих в газоконденсатной залежи, признано композиционное моделирование, результатом которого является модель пластового флюида. В рамках данного исследования моделирование производилось в программном комплексе ECLIPSE (PVTi \& ECLIPSE Compositional) [9]. Исходными данными для модели послужили сведения о составе, физико-химических и термодинамических свойствах пластового флюида, полученные на основе специальных промысловых и лабораторных исследований газоконденсата. Однако в связи с тем, что может присутствовать фактор неопределенности, обусловленный возможными неточностями и ошибками при проведении замеров, было уделено особое внимание выбору максимально представительной пробы, на основе которой рассчитывается композиционная модель, с целью уменьшений искажений в оценках фазового состояния и свойств компонентов флюида при создании модели газожидкостной смеси $[10,11]$.

В рамках исследования была использована наиболее представительная для исследуемого месторождения $\mathrm{N}$ проба газа и газового конденсата, свойства которой представлены в табл. 1.

Таблица 1. Параметры выбранной для моделирования nробы

Table 1. Parameters of sample simulation

\begin{tabular}{|c|c|c|c|}
\hline $\begin{array}{l}\text { Параметр } \\
\text { Parameter }\end{array}$ & $\begin{array}{c}\text { Обозначение } \\
\text { Designation }\end{array}$ & $\begin{array}{c}\text { Значение } \\
\text { Value }\end{array}$ & $\begin{array}{l}\text { Единица измерения } \\
\text { Unit of measurement }\end{array}$ \\
\hline $\begin{array}{l}\text { Пластовое } \\
\text { давление } \\
\text { Formation pressure }\end{array}$ & $P_{\text {пл }}$ & 256 & бар \\
\hline $\begin{array}{l}\text { Пластовая темпе- } \\
\text { paтура } \\
\text { Formation temper- } \\
\text { ature }\end{array}$ & $T_{\text {пл }}$ & 32 & ${ }^{\circ} \mathrm{C}$ \\
\hline $\begin{array}{l}\text { Давление начала } \\
\text { конденсации } \\
\text { Dew point pressure }\end{array}$ & $P_{\text {нк }}$ & 242 & бар \\
\hline $\begin{array}{l}\text { Конденсато- } \\
\text { газовый фактор } \\
\text { Condensate-gas } \\
\text { factor } \\
\end{array}$ & $К Г \Phi$ & 321,8 & $\mathrm{~cm}^{3} / \mathrm{m}^{3}$ \\
\hline $\begin{array}{l}\text { Плотность } \\
\text { конденсата } \\
\text { Density }\end{array}$ & $\rho_{\text {конд }}$ & 705 & $\kappa \Gamma / \mathrm{M}^{3}$ \\
\hline
\end{tabular}

Построенная тернарная диаграмма, представленная на рис. 2, отображает состав и фазовое состояние флюида в условиях начального пластового давления и 
температуры. Двухфазное состояние смеси при заданных термобарических условиях $\left(P_{\text {пл}}, T_{\text {пл }}\right)$ иллюстрируется закрашенной областью на диаграмме. Однако точка, характеризующая состав выбранной пробы флюида, лежит вне этой области, что говорит об однофазном газообразном состоянии пластового флюида в момент начала разработки $[12,13]$.

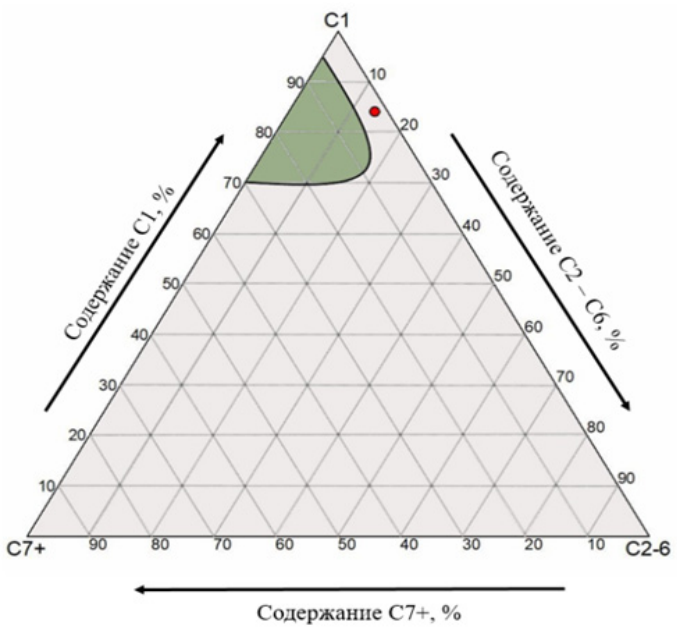

Рис. 2. Тернарная диаграмма пластовой смеси

Fig. 2. Ternary diagram of formation fluid

Для построения корректной модели углеводородной смеси, которая будет полностью характеризовать исходный пластовый флюид и его фазовое поведение, необходимо не только задать состав и свойства флюида, но и настроить PVT-модель, то есть правильно определить параметры уравнения состояния на основе принципов парожидкостного равновесия фаз при конкретных термобарических условиях и фактическом составе пластового газа (табл. 2), газа сепарации и газового конденсата [14-16].

Таблица 2. Компонентный состав пластового флюида Table 2. Formation fluid composition

\begin{tabular}{|c|c|c|}
\hline $\begin{array}{c}\text { Компонент } \\
\text { Component }\end{array}$ & $\begin{array}{c}\text { Содержание, \% } \\
\text { Content, } \%\end{array}$ & $\begin{array}{c}\text { Молярная масса, г/моль } \\
\text { Molar mass, } \mathrm{g} / \mathrm{mol}\end{array}$ \\
\hline $\mathrm{N} 2$ & 3,768 & 28,013 \\
\hline $\mathrm{He}$ & 0,188 & 4,0 \\
\hline $\mathrm{C}_{1}$ & 79,066 & 16,04 \\
\hline $\mathrm{C}_{2}$ & 6,747 & 30,07 \\
\hline $\mathrm{C}_{3}$ & 2,085 & 44,097 \\
\hline $\mathrm{C}_{4}$ & 1,679 & 58,124 \\
\hline $\mathrm{C}_{5+}$ & 4,511 & 76,341 \\
\hline $\mathrm{C}_{7+}$ & 1,128 & 105,87 \\
\hline $\mathrm{C}_{10+}$ & 0,828 & 166,57 \\
\hline
\end{tabular}

При создании композиционной модели газоконденсатной пластовой смеси использовался алгоритм, описанный в работе Т.С. Ющенко [17]. Настройка PVTмодели выполнена на основе результатов следующих лабораторных исследований пластового флюида.

1. Dew point test (определение давления начала конденсации).

2. Separator test (ступенчатая сепарация, определение плотности и газового фактора с учетом стандартных условий на последней ступени сепарации) [18].
3. Constant Composition Expansion test (CСЕ, исследования при постоянной массе), благодаря которому определяется PV соотношение, z-фактор газа, плотность и вязкость пластового флюида, объем жидкой фазы и давление насыщения.

4. Constant Volume Depletion test (CVD, исследования при постоянном объеме), которое позволяет определить z-фактор газа, объем насыщенной жидкой фазы, состав и свойства газа и выделившегося конденсата на каждой ступени сепарации.

Так как корректность PVT-модели газового конденсата в значительной степени определяется свойствами группы углеводородов $\mathrm{C}_{5+}$, то для повышения ее достоверности и точности было проведено искусственное разбиение группы углеводородов (УВ) $\mathrm{C}_{5+}$ на несколько псевдофракций методом, предложенным C.H. Whitson $[19,20]$, который основан на предположении, что распределение мольных долей тяжёлых фракций описывается гамма-распределением. В нашем случае разбиение производилось на 30 компонентов с последующей группировкой до $\mathrm{C}_{5+}, \mathrm{C}_{7+}$ и $\mathrm{C}_{10+}$ с целью корректирования свойств флюида и дальнейшей группировки до необходимого количества компонентов.

Таким образом, созданная композиционная модель флюида позволяет достаточно точно прогнозировать значения таких параметров, как давление насыщения, плотность, вязкость, объёмный коэффициент и фазовое состояние пластового флюида как в пластовых условиях, так и на ступенях сепарацุиน.

\section{Геологическое моделирование}

При построении геологической модели производится агрегация всей имеющейся геологической информации о залежи, в том числе о запасах УВ. Геологическое моделирование является основой для последующего гидродинамического моделирования процесса разработки залежи [21]. Для проведения сравнительного анализа режимов разработки создана упрощенная геологическая модель с принятыми для данной залежи усредненными геолого-геофизическими параметрами продуктивного интервала, представленными в табл. 3 . Неоднородность залежи по проницаемости для решения поставленной задачи по оценке эффективности разных режимов разработки с применением сайклинга достаточно описать коэффициентом анизотропии, который связан с процессами осадконакопления в период формирования породы-коллектора и который принят равным $k_{\text {пр.в }} / k_{\text {пр.г }}=0,15$. Помимо этого, породы, слагающие исследуемую залежь, характеризуются межзерновой пористостью, которая в модели принята одинаковой для всего продуктивного интервала вследствие ее малой изменчивости по объему.

Объемы геологических запасов каждого компонента в стандартных условиях, заключённых в созданной модели, рассчитывались по формуле:

$$
V_{i}=\frac{Z_{i} \times\left(G R V \times K_{\mathrm{\Pi}} \times\left(1-K_{\mathrm{BO}}-K_{\mathrm{HO}}\right)\right)}{B_{0}},
$$


где $V i$ - геологические запасы $i$-го компонента; $Z i-$ мольная доля $i$-го компонента в смеси (рис. 1); $G R V=2000 * 2000 / 10=40 * 106$ м $^{3}$ - объем куба (модели); $K_{\Pi}=0,12$ - коэффициент пористости; $K_{\text {во }}=0,12$ - остаточная водонасыщенность; $K_{\text {но }}=0,32$ - остаточная нефтенасыщенность; $\mathrm{B}_{0}$ - объемный коэффициент газа с учетом $z$-фактора.

Таблица 3. Параметры геологической модели

Table 3. Parameters of geologic model

\begin{tabular}{|c|c|c|c|}
\hline $\begin{array}{l}\text { Параметр } \\
\text { Parameter }\end{array}$ & $\begin{array}{c}\text { Обозначение } \\
\text { Designation }\end{array}$ & $\begin{array}{l}\text { Значение } \\
\text { Value }\end{array}$ & \begin{tabular}{|c|} 
Единица \\
измерения \\
Unit of \\
measurement
\end{tabular} \\
\hline $\begin{array}{l}\text { Размер модели } \\
\text { (Model size) }\end{array}$ & $\mathrm{X}, \mathrm{Y}, \mathrm{Z}$ & $2000 \times 2000 \times 10$ & м \\
\hline Пористость (Porosity) & $K_{\text {п }}$ & 12 & $\%$ \\
\hline $\begin{array}{l}\text { Горизонтальная про- } \\
\text { ницаемость } \\
\text { (Horizontal } \\
\text { permeability) }\end{array}$ & $K_{\text {пр.г }}$ & 100 & мД \\
\hline $\begin{array}{l}\text { Вертикальная прони- } \\
\text { цаемость } \\
\text { (Vertical permeability) }\end{array}$ & $K_{\text {пр.в }}$ & 15 & мД \\
\hline $\begin{array}{l}\text { Песчанистость } \\
\text { (Net to gross) }\end{array}$ & $N T G$ & 0,8 & Ед. \\
\hline $\begin{array}{l}\text { Остаточная } \\
\text { водонасыщенность } \\
\text { (Residual water } \\
\text { saturation) }\end{array}$ & $K_{\text {во }}$ & 12 & $\%$ \\
\hline $\begin{array}{l}\text { Остаточная } \\
\text { нефтенасыщенность } \\
\text { (Residual oil } \\
\text { saturation) }\end{array}$ & $K_{\text {но }}$ & 32 & $\%$ \\
\hline
\end{tabular}

\section{Гидродинамическое моделирование}

На основе геологической модели была построена гидродинамическая модель объекта исследуемой залежи, которая позволяет не только прогнозировать технологические показатели разработки, но и является инструментом для принятия производственных решений [16]. Данная модель является инструментом визуализации процессов, происходящих в залежи с заданными геолого-физическими свойствами (табл. 3) и насыщенной углеводородной смесью, состав и свойства которой описаны посредством композиционного моделирования (табл. 1, 2). Гидродинамическая модель позволяет дать оценку эффективности применения сайклинг-технологии в условиях газоконденсатной залежи путем прогнозирования показателей разработки на 30 лет. В качестве инструмента для моделирования был использован гидродинамический симулятор tNavigator ${ }^{\circledR}$ компании Rock Flow Dynamics ${ }^{\circledR}$ (RFD), посредством которого была инициализирована цифровая секторная гидродинамическая модель газоконденсатной залежи N-го месторождения Восточной Сибири (рис. 3).

Модельный сектор является частью пятиточечной системы разработки и содержит две вертикальные скважины с перфорацией по всей эффективной толщине пласта. В рамках исследования сайклинг реализуется посредством нагнетания в пласт сухого газа, собранного с последней ступени сепарации, что позволяет решить проблему утилизации газа, а подбор низкотемпературного сепаратора предотвращает обратную закачку в пласт высококипящих компонентов.

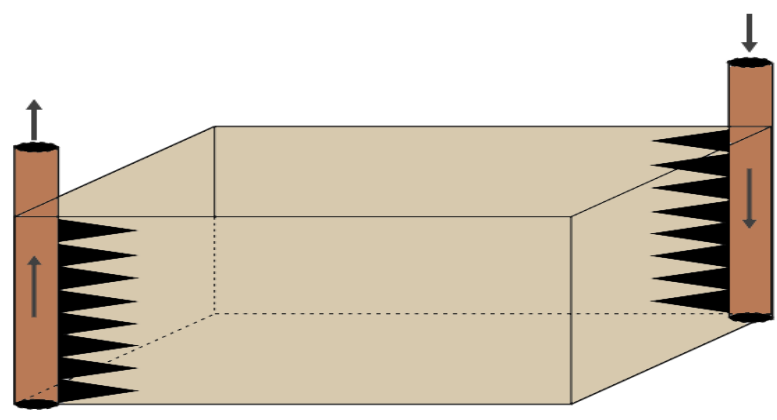

Рис. 3. Схема секторной гидродинамической модели залежи Fig. 3. Scheme of the sectoral hydrodynamic formation model

\section{Сравнительный анализ}

В настоящее время разработка газоконденсатных месторождений в России ведется, как правило, без поддержания пластового давления, то есть на режиме истощения.

При разработке газоконденсатных месторождений особое внимание уделяется в первую очередь извлечению непосредственно газового конденсата, содержащего углеводородную группу компонентов $\mathrm{C}_{5+}$. Однако нельзя забывать о том, что большую часть природного газа составляют именно легкие УВ, которые также являются важным энергетическим ресурсом и их добыча приносит добывающим компаниям значительный экономический эффект. Поэтому при выборе оптимального сценария разработки необходимо учитывать покомпонентную добычу УВ $\left(\mathrm{C}_{1}, \mathrm{C}_{2}\right.$, $\mathrm{C}_{3}-\mathrm{C}_{4}, \mathrm{C}_{5_{+}}$). В связи с этим критерием эффективности того или иного режима разработки выбран такой показатель, как коэффициент извлечения компонента (КИК), т. е. отбор компонента в зависимости от его геологических запасов.

Так ли эффективен сайклинг и есть ли необходимость в его применении? Для ответа на эти вопросы проведен сравнительный анализ эффективности разработки месторождения на режиме истощения и с применением сайклинга. На рис. 4 приведены дебиты газового конденсата со скважины исследуемого месторождения с прогнозом на 30 лет для указанных выше режимов.

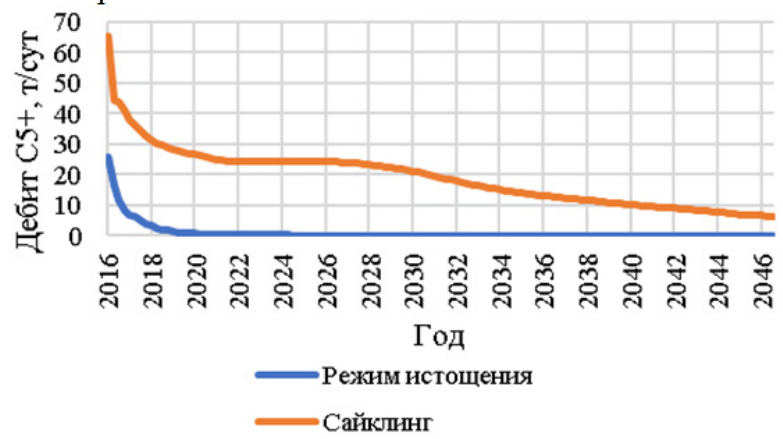

Puc. 4. Дебить газового конденсата в динамике на режимах истощения и с применением сайклинга

Fig. 4. Flow rates of gas condensate in dynamics at depletion drive and cycling 
Коэффициент извлечения конденсата при работе на режиме истощения составляет $23 \%$, на режиме сайклинга - $62 \%$, что объясняется поддержанием Рпл закачкой в пласт сухого газа и предотвращением потерь компонентов $\mathrm{C}_{5+}$ вследствие процессов ретроградной конденсации. Отсюда можно сделать вывод о том, что применение сайклинга для разработки газоконденсатной залежи увеличивает извлечение конденсата в три раза и является эффективным технологическим решением.

\section{Повышение эфффективности сайклинг-технологии}

Целью дальнейших исследований стал поиск способов повышения эффективности сайклинга, в рамках которого эмпирическим методом производился подбор следующих параметров: 1) время начала воздействия на пласт для максимального извлечения компонентов; 2) оптимальные объемы закачки (коэффициент компенсации); 3) порог рентабельности технологии.

В работе смоделированы и рассчитаны 10 режимов разработки газоконденсатной залежи, критериями эффективности которых выбраны КИК и темпы отбора газового конденсата. Моделирование сайклинга производилось на компенсации в 50 и $100 \%$ и с нагнетанием газа в объемах, равных максимальной приемистости газонагнетательной скважины (рис. 5).

Режим истощения с последующим сайклингом подразумевает введение системы поддержания Рпл путем закачки сухого газа через какое-то время после начала разработки. Такой сценарий разработки основан на предварительном истощении залежи, в процессе которого вырабатываются в основном запасы низкокипящих компонентов, после чего запускается сайклинг для извлечения газового конденсата.

Режимы с переходом на истощение после сайклинга обоснованы необходимостью извлекать уже сухой газ после того, как запасы конденсата отработаны [22]. Помимо этого, на эффективность сайклинга большое влияние оказывает коэффициент компенсации, который равен отношению объемов закачиваемого и добываемого газа в пластовых условиях и напрямую зависит от доступного количества реагента закачки, технических мощностей и т. д.

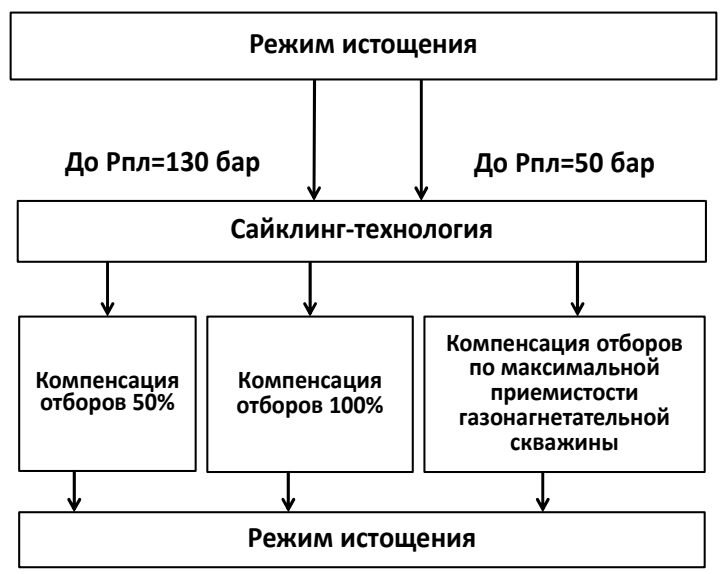

Pис. 5. Режимы разработки газоконденсатной залежи

Fig. 5. Gas condensate reservoir development mechanisms

По результатам гидродинамического моделирования построена гистограмма дебитов газового конденсата (рис. 6), из которой можно сделать вывод о том, что варианты с предварительным истощением являются малоэффективными для извлечения компонентов $\mathrm{C}_{5+}$. Такой результат объясняется стремительным снижением Рпл до уровня ниже Рнк до момента введения сайклинга, что ведет к ретроградной конденсации, а следовательно, и к снижению добычи газового конденсата.

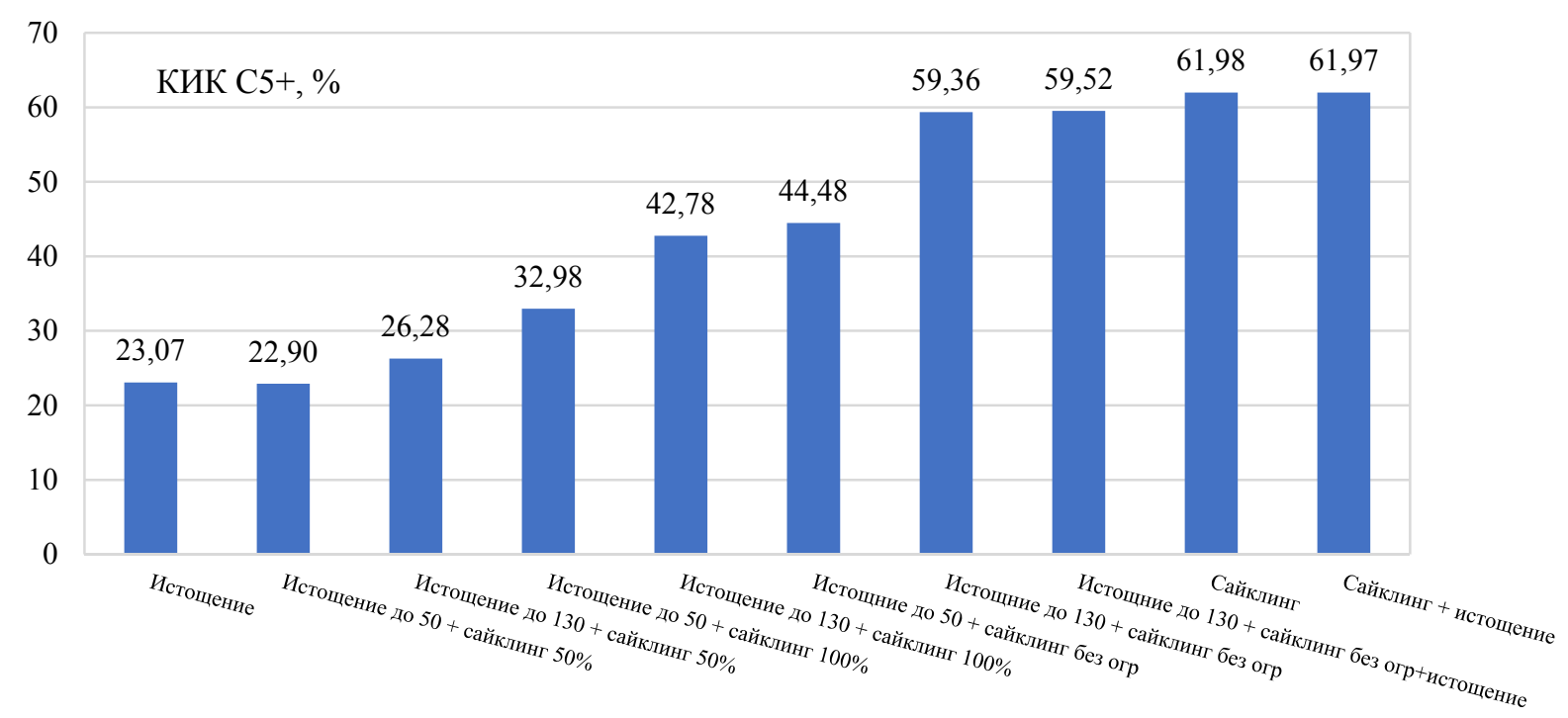

Pис. 6. Сравнительная гистограмма дебитов и коэффичиентов извлечения газового конденсата при различных комбинаииях режимов истощения и сайклинга

Fig. 6. Comparative histogram of flow rates and gas condensate recovery factor at different combinations of depletion drive and cycling 
Величина, на которую поднимется Рпл при подключении нагнетательной скважины, напрямую зависит от количества закачиваемого газа [23], поэтому более высокие коэффициенты извлечения конденсата достигаются при компенсации $100 \%$, чем при $50 \%$. Однако необходимо учесть, что доведение до $100 \%$ компенсации может быть не реализовано в силу необходимости подключения дополнительных объемов газа извне, что не всегда является возможным. Для режимов, на которых получены максимальные дебиты и, соответственно, коэффициенты извлечения конденсата, построена гистограмма добычи компонентов УВ порядка $\mathrm{C}_{1}-\mathrm{C}_{4}$ (рис. 7).

Для визуализции темпов отбора газового конденсата проведен анализ конденсато-газового фактора (КГФ) в добываемом флюиде, результаты которого представлены на рис. 8. Явление ретроградной конденсации при снижении Рпл ниже уровня Рнк приводит к осушению скважиной продукции посредством выпадения в пласте компонентов $\mathrm{C}_{5+}$ [24], о чем говорит низкий КГФ при истощении и более высокий при сайклинге.

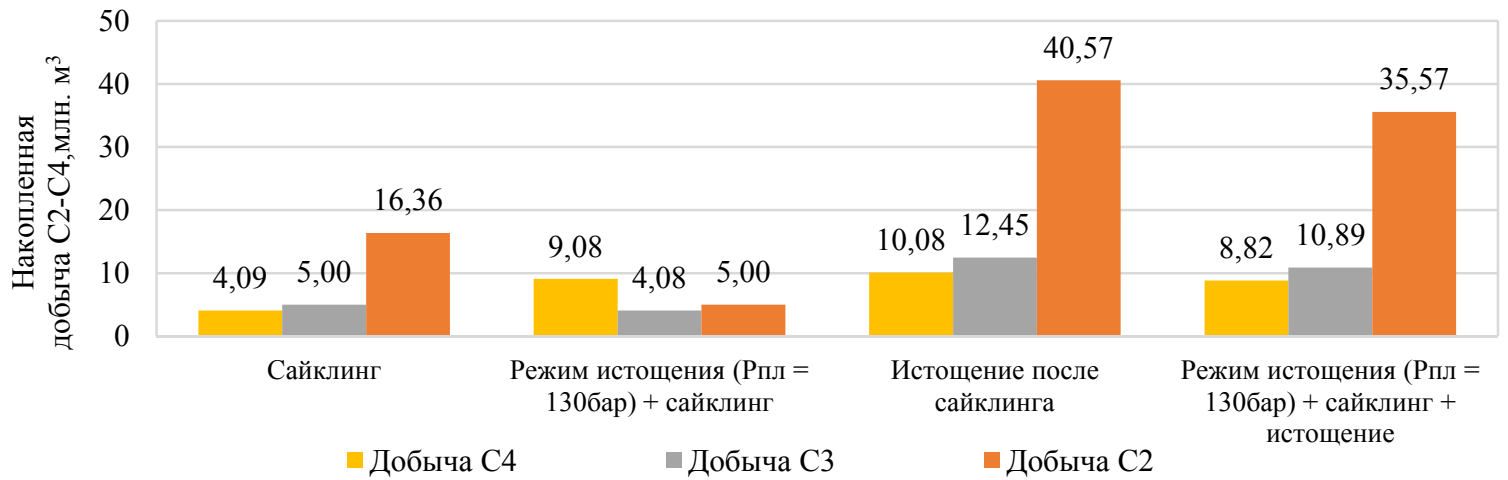

Pис. 7. Гистограмма накопленной добычи компонентов углеводородов $C_{1}$ - $C_{4}$

Fig. 7. Histogram of cumulative production of hydrocarbon component $C_{1}-C_{4}$

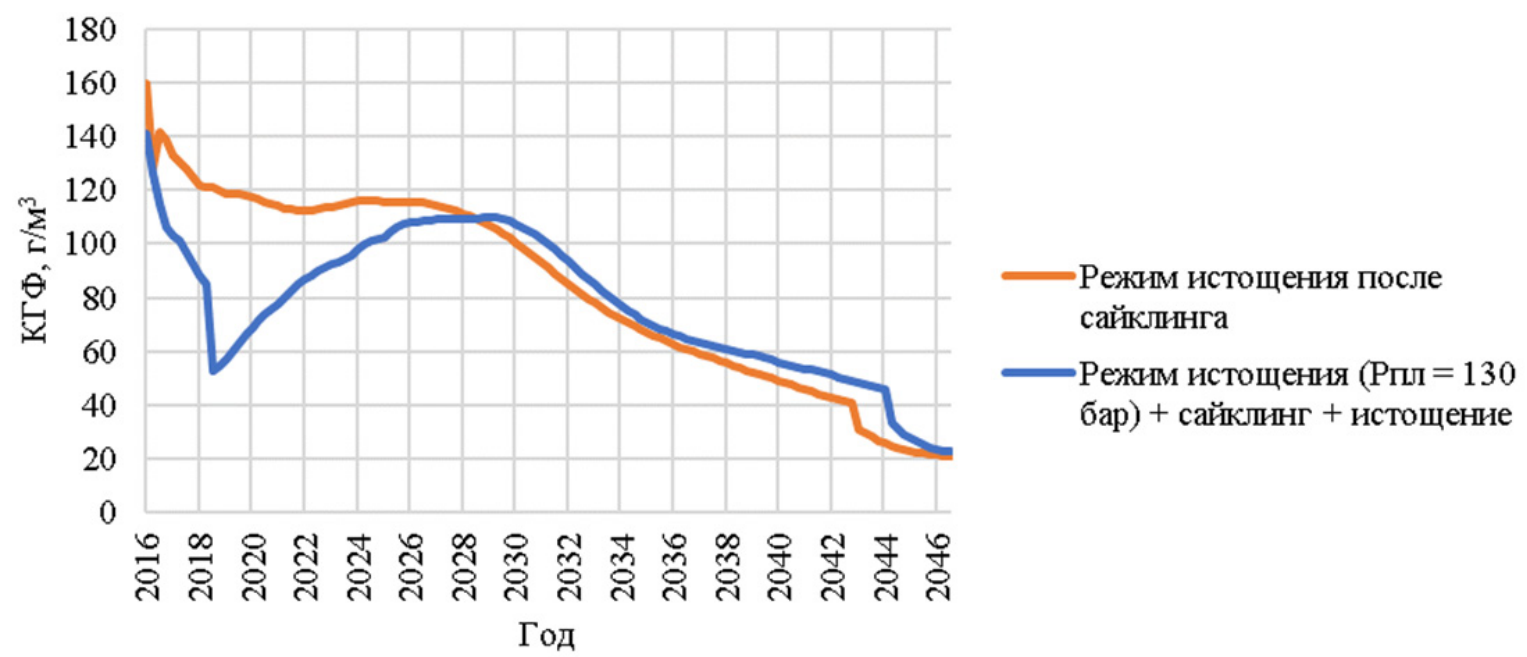

Puc. 8. Конденсато-газовый фактор в динамике, г/м

Fig. 8. Condensate-gas factor in dynamics, $\mathrm{g} / \mathrm{m}^{3}$

В случае разработки по сценарию «истощение, сайклинг, истощение» неизбежны значительные потери газового конденсата в первые годы разработки. Таким образом, высокие темпы отбора конденсата можно получить только путем поддержания пластового давления непосредственно с начала эксплуатации, не опуская его ниже давления начала конденсации газа

Как говорилось ранее, для экономической эффективности проекта необходимо учитывать добычу не только конденсата, но и других групп УВ. Истощение залежи после выработки газового конденсата имеет положительный эффект и позволяет извлекать низко- кипящие углеводородные компоненты, которые при дальнейшей оптимизации можно перевести в разряд товарных продуктов в виде СПГ, этилена и природного газа. Поэтому на последней стадии разработки газоконденсатного месторождения можно повысить эффективность проекта путем перевода нагнетательных скважин в добывающий фонд.

Проведенное технико-экономическое обоснование показывает, что высокий темп отбора конденсата и низкокипящих УВ именно в первые годы разработки месторождения приносит максимальный экономический эффект. 


\section{Заключение}

Для решения проблемы, связанной с потерями высококипящих компонентов группы УВ группы $\mathrm{C}_{5+}$ при разработке газоконденсатных залежей, предложено применение сайклинг-технологии, которая позволяет:

- предотвратить выпадение конденсата в пласте путем поддержания пластового давления;

- обеспечить максимальный отбор газового конденсата в первые годы разработки;

- обеспечить эффективную разработку залежи при условии 100 \% компенсации отборов из пласта;

- решить экологические проблемы, связанные с утилизацией попутного газа;

- осуществлять отбор не только УВ группы $\mathrm{C}_{5+}$, но и этана, пропана и бутана.

Проведенные расчеты на основе усовершенствованной композиционной модели газоконденсатной смеси и секторной геологической модели залежи показали, что применение сайклинга для разработки

\section{СПИСОК ЛИТЕРАТУРЫ}

1. Tarek A. Reservoir engineering handbook. - London, UK: Elsevier Science \& Technology, Gulf Professional Publ., 2010. - 1463 p.

2. Al-Baqawi A.M., Al-Malki B.H. Well test analysis in naturally fractured gas condensate reservoirs below dew point pressure // Asia Pacific Oil and Gas Conference and Exhibition. - Jakarta: Society of Petroleum Engineers, 2009. URL: https://www.onepetro.org/conference-paper/SPE-122594-MS (дата обращения 03.09.2019).

3. Siddiqui M.A.Q., Alnuaim S., Khan R.A. Well placement and rate optimization for gas cycling in gas condensate reservoirs // SPE Middle East Oil \& Gas Show and Conference. - Manama, Bahrain Society of Petroleum Engineers, 2015. URL: https://www.onepetro.org/conference-paper/SPE-172641-MS (дата обращения 03.09.2019).

4. Закономерности исчерпания запасов нефти и газа в России и прогноз их воспроизводства / И.В. Филимонова, Л.В. Эдер, И.В. Проворная, А.В. Комарова // Экологический вестник России. - 2018. - № 4. - С. 4-12.

5. Current state and problems of integrated development of mineral resources base in Russia / I.V. Filimonova, L.V. Eder, M.V. Mishenin, T.M. Mamakhatov // IOP Conference Series: Earth and Environmental Science. - 2017. - V. 84. - № 1. - P. 1-5.

6. Key problems in the development of the power of Siberia project / A.E. Kontorovich, L.V. Eder, I.V. Filimonova, S.M. Nikitenko // Regional Research of Russia. - 2018. - V. 8. - № 1. - P. 92-100

7. Эдер Л.В., Проворная И.В., Филимонова И.В. Проблемь рационального использования попутного нефтяного газа в России // География и природные ресурсы. - 2019. - Т. 40. № 1. - C. 9-14.

8. Sharf I., Tsibulnikova M., Dmitrieva N. Economic evaluation of the approaches to associated petroleum gas utilization // Ecology, Economics, Education and Legislation: Proc. 16th International multidisciplinary scientific geoconference (SGEM 2016). - Sofia: STEF92 Technology Ltd, 2016. - V. 2-5. - P. 153-160.

9. PVTi and ECLIPSE 300. An Introduction to PVT analysis and compositional simulation. - Houston, USA: Schlumberger, Abingdon Technology Center Training 2005. - $402 \mathrm{p}$.

10. Щебетов А.В., Галкин М.В. Оценка качества и моделирование газоконденсатных исследований в условиях неопределенности исходных данных // Газовая промышленность. - 2009. № 9. - C. 40-44.

11. Научные основы прогноза фазового поведения пластовых газоконденсатных систем // А.И. Гриценко, И.А. Гриценко, В.В. Юшкин, Т.Д. Островская. - М.: Недра, 1995. - 432 с.

12. Afidick D., Kaczorowski N.J., Bette S. Production performance of retrograde gas reservoir: a case study of the Arun field // SPE Asia газоконденсатной залежи увеличивает извлечение конденсата в три раза на прогнозируемом периоде в 30 лет.

В результате модельных расчетов получен коэффициент извлечения конденсата, равный $62 \%$, и высокий конденсатно-газовый фактор в первые 10 лет разработки. Также выделен наиболее оптимальный режим разработки газоконденсатной залежи, который предполагает применение режима истощения после реализации сайклинга.

Авторы считают возможным тиражирование технологии сайклинга на месторождениях Западной Сибири путем адаптации модели под необходимые геологические условия.

Работа выполнена при финансовой поддержке РФФИ, грант 18-010-00660 А Конщептуальные подходы к парадигме устойчивого и сбалансированного недропользования области с учетом специфики минерально-сырьевой базы и отраслевой структуры в целях обеспечения долгосрочного социально-экономического роста нефтедобывающего региона.

Pacific Oil and Gas Conference. - Melbourne: The Society Publ., 1994. - P. 73-80.

13. Брусиловский А.И. Фазовые превращения при разработке месторождений нефти и газа. - М.: Грааль, 2002. -575 с.

14. Грачев С.И., Краснова Е.И. Термодинамические процессы при разработке нефтегазоконденсатных месторождений. - Тюмень: ТюмГНГУ, 2015. $-99 \mathrm{c}$

15. Брусиловский А.И. Моделирование фазового состояния и термодинамических свойств природных многокомпонентных систем при проектировании разработки и эксплуатации месторождений нефти и газа: дис. ... д-ра техн. наук. - М., 1994. - 602 c.

16. Spivak A., Dixon T.N. Simulation of gas-condensate reservoirs // SPE Symposium on Numerical Simulation of Reservoir Performance. - Houston, USA: Society of Petroleum Engineers, 1973. URL: https://www.onepetro.org/conference-paper/SPE4271-MS (дата обращения 03.09.2019).

17. Ющенко Т.С. Методика автоматизированного создания термодинамических моделей газоконденсатных пластовых углеводородных систем на основе кубического уравнения состояния // Труды МФТИ. - 2014. - Т. 6. - № 3. - С. 154-161.

18. Шарипов А.Ф., Волков А.Н. Анализ критериев оценки качества отбора проб при газоконденсатных исследованиях // Вестник ЦКР Роснедра. - 2015. - № 4. - С. 33-41.

19. Use of compositional simulation in the management of Arun gas condensate reservoir / T. Sutan-Assin, S.C. Rastogi, M. Abdullah, D. Hidayat // 7th Offshore South East Asia Conference. Singapore: Society of Petroleum Engineers, 1988. URL: https://www.onepetro.org/conference-paper/SPE-17683-MS (дата обращения 05.09.2019).

20. Whitson C.H., Brule M.R. Phase behavior. - Texas: Richardson, 2000. $-240 \mathrm{p}$.

21. Итерационный подход при геологическом и гидродинамическом моделировании / Д.А. Зундэ, А.А. Горланов, И.П. Шаламов, А.И. Давлетшин, В.Ю. Белянский, О.А. Лознюк, К.Е. Закревский, В.Н. Абрашов // Нефтяное хозяйство. - 2019. - № 5. - С. 58-61.

22. Dake L.P. Fundamentals of reservoir engineering. - Amsterdam, The Netherlands: Elsevier science B.V, 1978. -438 p.

23. Aziz R.M. Critique on gas cycling operations in gas condensate reservoirs // Middle East Oil Technical Conference and Exhibition. - Manama, Bahrain: Society of Petroleum Engineers, 1983. URL: https://www.onepetro.org/conference-paper/SPE-11477-MS (дата обращения 05.09.2019).

24. Инструкция по комплексному исследованию газовых и газоконденсатных пластов и скважин / под ред. Г.А. Зотова, 3.С. Алиева. - М.: Недра, 1980. - 301 с.

Поступила 14.11.2019 2. 


\section{Информация об авторах}

Волжженина Д.А., магистрант отделения нефтегазового дела Инженерной школы природных ресурсов Национального исследовательского Томского политехнического университета.

Шарф И.В., кандидат экономических наук, доцент отделения нефтегазового дела Инженерной школы природных ресурсов Национального исследовательского Томского политехнического университета.

Сабанчин И.В., директор департамента разработки месторождений Иркутской Нефтяной Компании. 
UDC 87.35 .91

\title{
ANALYSIS OF EFFICIENCY OF APPLYING CYCLING IN RESERVOIR DEVELOPMENT OF GAS CONDENSATE
}

\author{
Diana A. Volzhenina1, \\ Volzhenina_DA@irkutskoil.ru \\ Irina V. Sharf', \\ irina_sharf@mail.ru \\ Igor V. Sabanchin2, \\ isabanchin@irkutskoil.ru \\ 1 National Research Tomsk Polytechnic University, \\ 30, Lenin avenue, Tomsk, 634050, Russia. \\ 2 Irkutsk Oil Company, \\ 4, Bolshoy Liteiny avenue, Irkutsk, 664007, Russia.
}

The relevance of the research is conditioned by the processes of retrograde condensation while developing gas-condensate reservoirs at oil and gas fields. Due to these processes the reservoir losses its significant volumes of high-boiling components of $\mathrm{C}_{5+}$ hydrocarbon group, the production of which would be an additional income source for oil companies. The total reserves of gas condensate in Russia is about two billion tons. Therefore, to solve the problem of the fullest extraction of gas-condensate reservoir components it is necessary to implement new production methods.

The research aim is to justify the efficiency of cycling technology in gas-condensate reservoir development.

Subject: $N$ field located in the territory of the Eastern Siberia.

Methods: compositional, geologic, and hydrodynamic simulation of gas-condensate reservoir

Results. The paper presents the results of gas-condensate formation fluid compositional simulation in accordance with gas and condensate sample composition as well as gas-condensate study. Simplified geologic model of studied reservoir has been built and hydrodynamic simulation of its condition has been performed for different recovery mechanisms. Comparative analysis of gas condensate reservoir development parameters (condensate recovery factor, $\mathrm{C}_{2}-\mathrm{C}_{4}$ recovery factor, and condensate-gas factor) at different recovery mechanisms proved that application of cycling results in increase of extraction of hydrocarbon group $\mathrm{C}_{5+}$ component and allows solving a number of economic and environmental problems. It is possible to adapt the model to the required geologic conditions to use the given technology in the territory of Eastern Siberia and other petroleum provinces.

\section{Key words:}

Reservoir, development, high-boiling hydrocarbon groups, cycling, gas-condensate reservoir, compositional, geologic, and hydrodynamic simulation, condensate recovery factor.

The research was financially supported by the RFBR, grant 18-010-00660 A Conceptual approaches to the paradigm of stable and balanced subsurface management of the region considering the specific character of mineral resource base and sectoral structure to support long-term social and economic growth of oil producing region.

\section{REFERENCES}

1. Tarek A. Reservoir engineering handbook. London, UK, Elsevier Science \& Technology, Gulf Professional Publ., 2010. 1463 p.

2. Al-Baqawi A.M., Al-Malki B.H. Well test analysis in naturally fractured gas condensate reservoirs below dew point pressure. Asia Pacific Oil and Gas Conference and Exhibition. Jakarta, Society of Petroleum Engineers, 2009. Available at: https://www.onepetro.org/conference-paper/SPE-122594-MS (accessed 3 September 2019).

3. Siddiqui M.A.Q., Alnuaim S., Khan R.A. Well placement and rate optimization for gas cycling in gas condensate reservoirs. SPE Middle East Oil \& Gas Show and Conference. Manama, Bahrain: Society of Petroleum Engineers, 2015. Available at: https://www.onepetro.org/conference-paper/SPE-172641-MS (accessed 3 September 2019)

4. Filimonova I.V., Eder L.V., Provornaya I.V., Komarova A.V. Regularities of oil and gas reserve depletion in Russia and the forecast of their reproduction. Environmental bulletin of Russia, 2018, no. 4, pp. 4-12. In Rus.

5. Filimonova I.V., Eder L.V., Mishenin M.V., Mamakhatov T.M. Current state and problems of integrated development of mineral resources base in Russia. IOP Conference Series: Earth and Environmental Science, 2017, vol. 84, no. 1, pp. 1-5.
6. Kontorovich A.E., Eder L.V., Filimonova I.V., Nikitenko S.M. Key problems in the development of the power of Siberia project. Regional Research of Russia, 2018, vol. 8, no. 1, pp. 92-100.

7. Eder L.V., Provornaya I.V., Filimonova I.V. Problems of rational use of associated petroleum gas in Russia. Geography and Natural Resources, 2019, vol. 40, no. 1, pp. 9-14. In Rus.

8. Sharf I., Tsibulnikova M., Dmitrieva N. Economic evaluation of the approaches to associated petroleum gas utilization. Ecology, Economics, Education and Legislation: Proc. $16^{\text {th }}$ International multidisciplinary scientific geoconference (SGEM 2016). Sofia, STEF92 Technology Ltd, 2016. Vol. 2-5, pp. 153-160.

9. PVTi and ECLIPSE 300. An Introduction to PVT analysis and compositional simulation. Houston, USA, Abingdon Technology Center Training, Schlumberger, 2005. $402 \mathrm{p}$.

10. Shhebetov A.V., Galkin M.V. Otsenka kachestva i modelirovanie gazokondensatnykh issledovaniy $\mathrm{v}$ usloviyakh neopredelennosti iskhodnykh dannykh [Quality assessment and modeling of gas condensate studies under uncertainty of initial data]. GAS Industry of Russia, 2009, no. 9, pp. 40-44.

11. Gritsenko A.I., Gritsenko I.A., Yushkin V.V., Ostrovskaya T.D. Nauchnye osnovy prognoza fazovogo povedeniya plastovykh gazokondensatnykh sistem [Scientific bases for prediction of phase behavior of reservoir gas condensate systems]. Moscow, Nedra Publ., 1995. $432 \mathrm{p}$. 
12. Afidick D., Kaczorowski N.J., Bette S. Production performance of retrograde gas reservoir: a case study of the Arun field. SPE Asia Pacific Oil and Gas Conference. Melbourne, The Society Publ., 1994. pp. 73-80.

13. Brusilovskiy A.I. Fazovye prevrashcheniya pri razrabotke mestorozhdeniy nefti i gaza [Phase transformations in oil and gas field development]. Moscow, Graal Publ., 2002. 575 p.

14. Grachev S.I., Krasnova E.I. Termodinamicheskie protsessy pri razrabotke neftegazokondensatnykh mestorozhdeniy [Thermodynamic processes in the development of oil and gas condensate fields]. Tyumen, Industrial University of Tyumen Publ., 2015. $99 \mathrm{p}$.

15. Brusilovskiy A.I. Modelirovanie fazovogo sostovaniva $i$ termodinamicheskikh svoystv prirodnykh mnogokomponentnykh sistem pri proektirovanii razrabotki $i$ ekspluatatsii mestorozhdeniy nefti i gaza. Diss. Dokt. nauk [Modeling of the phase state and thermodynamic properties of natural multicomponent systems in the design development and operation of oil and gas fields. Dr. Diss.]. Moscow, 1994. $602 \mathrm{p}$.

16. Spivak A., Dixon T.N. Simulation of gas-condensate reservoirs SPE Symposium on Numerical Simulation of Reservoir Performance. Houston, USA, Society of Petroleum Engineers, 1973. Available at: https://www.onepetro.org/conference-paper/SPE4271-MS (accessed 3 September 2019).

17. Yushchenko T.S. Metodika avtomatizirovannogo sozdaniya termodinamicheskikh modeley gazokondensatnykh plastovykh uglevodorodnykh sistem na osnove kubicheskogo uravneniya sostoyaniya [Method of automated development of thermodynamic models of gas condensate reservoir hydrocarbon systems based on the cubic state equation]. Proceedings of Moscow Institute of Physics and Technology, 2014, vol. 6, no 3, pp. 154-161.
18. Sharipov A.F., Volkov A.N. Analysis of sampling quality assessment criteria during gas condensate studies. Vestnik CKR Rosnedra, 2015, no 4, pp. 33-41. In Rus.

19. Sutan-Assin T., Rastogi S.C., Abdullah M., Hidayat D. Use of compositional simulation in the management of Arun gas condensate reservoir. 7th Offshore South East Asia Conference. Singapore, Society of Petroleum Engineers, 1988. Available at: https://www.onepetro.org/conference-paper/SPE-17683-MS (accessed 5 September 2019).

20. Whitson C.H., Brule M.R. Phase behavior. Texas, Richardson, 2000. $240 \mathrm{p}$

21. Zunde D.A., Gorlanov A.A., Shalamov I.P., Davletshin A.I., Belyansky V.Yu., Loznyuk O.A., Zakrevskiy K.E., Abrashov V.N. Iterative approach to geological and hydrodynamic modeling. Oil Industry Journal, 2019, no. 5. In Rus. Available at: https://onepetro.org/journal-paper/OIJ-2019-05-058-061-RU (accessed 5 September 2019).

22. Dake L.P. Fundamentals of reservoir engineering. Amsterdam, The Netherlands, Elsevier science B.V, 1978. 438 p.

23. Aziz R.M. Critique on gas cycling operations in gas condensate reservoirs. Middle East Oil Technical Conference and Exhibition. Manama, Bahrain, Society of Petroleum Engineers, 1983. Available at: https://www.onepetro.org/conference-paper/SPE-11477-MS (accessed 5 September 2019).

24. Instruktsiva po kompleksnomu issledovaniyu gazovykh i gazokondensatnykh plastov $i$ skvazhin [Instructions for comprehensive study of gas and gas condensate reservoirs and wells]. Eds. G.A. Zotov, Z.S. Aliev. Moscow, Nedra Publ., 1980. 301 p.

Received: 14 November 2019.

\section{Information about the authors}

Diana A. Volzhenina, undergraduate, National Research Tomsk Polytechnic University.

Irina V. Sharf, Cand. Sc., associate professor, National Research Tomsk Polytechnic University. Igor V. Sabanchin, Director of the Field Development Department, Irkutsk Oil Company. 